\title{
Symmetric or not? A holistic approach to the measurement of fluctuating asymmetry from facial photographs
}

Selina Weiss ${ }^{1 * \#}$, C. Martin Grewe ${ }^{2 \# * *}$, Sally Olderbak ${ }^{1}$, Benjamin Goecke ${ }^{1}$, Laura Kaltwasser ${ }^{3}$, Andrea Hildebrandt ${ }^{4}$

\# The first two authors contributed equally to this manuscript.

${ }^{1}$ Department of Individual Differences and Psychological Assessment, Institute for Psychology and Education, Ulm University, Ulm, Baden-Wuerttemberg, Germany

${ }^{2}$ Department of Visual and Data-centric Computing, Zuse Institute Berlin (ZIB), Berlin, Germany

${ }^{3}$ Berlin School of Mind and Brain, Humboldt-Universität zu Berlin, Berlin, Germany

${ }^{4}$ Division of Psychological Methods and Statistics, Department of Psychology, Carl von Ossietzky Universität Oldenburg, Oldenburg, Niedersachsen, Germany

$* / * *$ Corresponding authors

E-mail addresses: selina.weiss@uni-ulm.de (S. Weiss), grewe@ zib.de (C.M. Grewe)

Author Note:

*Corresponding author at: Ulm University, Institute for Psychology and Pedagogy, Selina

Weiss, Albert-Einstein-Allee 47, 89081 Ulm, Germany. Telephone: +49 73150 31145. Fax: +49 731/50

31149. E-mail: selina.weiss@uni-ulm.de

** Corresponding author at: Zuse Institute Berlin (ZIB), Department of Visual and Data-centric

Computing, C. Martin Grewe, Takustraße 7, 14195, Berlin, Germany. Telefphone +49 3084185352.

Fax +49 3084185 107. E-mail: grewe@zib.de

This manuscript has been accepted for publication in Personality and Individual Differences in its current form. 


\title{
Highlights
}

- Existing asymmetry scores have limitations, e.g. correlation with head pose.

- Our new holistic score distinguishes between pose and various types of asymmetry.

- Attractiveness ratings correlate with pose but not with asymmetry.

- Perceived personality and femininity/masculinity correlate with asymmetry.

\begin{abstract}
Previous studies investigated the relationship between facial asymmetry, perceived attractiveness and peoples' traits. The diversity of results might be due to methodological issues and poor standardization between asymmetry scoring methods. We evaluate two traditional scoring methods, the Horizontal Angular Asymmetry (HAA) and the Horizontal Fluctuating Asymmetry (HFA), and propose a geometric morphometrics-based Holistic Facial Asymmetry Score (HFAS). In Study 1, 1000 photographs with different types of asymmetry were simulated and then correlated with the estimated facial asymmetry scores. Results indicate that HFA was highly affected by extrinsic factors, while HAA and HFAS were less affected and rather capture intrinsic asymmetry. In Study 2, HFAS and HAA were compared for 160 facial images (45\% Female, $M=27.6$ years) by estimating their correlations with ratings of attractiveness and targets' traits provided by 81 adults $(51 \%$ Female, $M=$ 27.7 years). Results suggest that attractiveness was only related with extrinsic factors. HAA was related with higher fairness, femininity, modesty, and sincerity, but less greediness and masculinity. HFAS was related with low femininity, high masculinity, and greediness, as well as low negativity, higher fairness, and sincerity. We discuss the benefits of HFAS as a scoring method for the assessment of facial asymmetry.
\end{abstract}

Keywords: Facial asymmetry; Traditional morphometrics; Geometric morphometrics; Trait perception; Face perception 


\section{Symmetric or not? A holistic approach to the measurement of fluctuating asymmetry from facial photographs}

\section{Introduction}

The left side of the human body is highly similar to its right side; the shapes of both sides are almost bilaterally symmetric (Palmer \& Strobeck, 1986). Asymmetry between the sides is purportedly caused by genetic factors and environmental stressors experienced throughout their development (Livshits \& Kobyliansky, 1989, Livshits \& Kobyliansky, 1991; Parsons, 1990; van Valen, 1962). Thus, high symmetry of the body and face reflect developmental stability while high asymmetry indicates stress and instability (e.g., Shackelford \& Larsen, 1997).

Over the last two decades, asymmetry of human faces has received increasing attention in the fields of anthropology (Bigoni et al., 2013), medicine (Thiesen, et al., 2015), and psychology (Svegar, 2016). In psychology, facial asymmetry is used as a biomarker of biological fitness and environmental stress with relevance for clinical, developmental, and social contexts, as well as personality (e.g., Hope et al., 2011; Shackelford \& Larsen, 1997). Facial asymmetry was found to vary within a person throughout their lifespan (Pound et al., 2014), as well as between persons at all stages of development (Thiesen et al., 2015). Facial asymmetry is related to self-reported personality traits (Borráz-León \& Cerda-Molina, 2015; Fink et al., 2005) and occurs in facial expressions of emotion (e.g., Carr et al., 2014). It correlates with external ratings of personality and attractiveness (e.g., Fink et al., 2006; Grammer \& Thornhill, 1994; Rhodes, 2006). Furthermore, facial asymmetry has been related to psychological, emotional, physiological distress (Shackelford \& Larsen, 1997), intelligence (Banks et al., 2010; Prokosch et al., 2004), and physical health (Pound, et al., 2014).

Although the majority of studies seem to support a positive relationship between facial asymmetry and health, there are disagreements in the magnitude, statistical significance, and even the sign of the correlation in regards to personality traits (Svegar, 2016). That is, the magnitude of the 
relation between attractiveness and facial asymmetry varies from negative to positive. A review that focuses on single physical features showed that across a variety of studies, facial asymmetry and attractiveness were not significantly related with one another (Weeden $\&$ Sabini, 2005). The heterogeneity of these results is attributed to the wide variety of measurement methods of all variables, especially to the scoring of facial asymmetry. The lack of standardization in (digital) data acquisition and scoring methods compromises the comparability of findings and limits evaluations of predictive validity (Banks et al., 2010). The present study investigates and compares different methods for the scoring of facial asymmetry from photographs and discusses their suitability for psychological research with a particular focus on perception and ratings of facial asymmetry.

\subsection{Challenges in facial asymmetry measurement}

Facial asymmetry accounts for shape differences between both sides of a face. The reference line of symmetry is the midsagittal, an ideal line or plane through the forehead, nose, mouth, and chin which separates both face halves. Bilaterally symmetric faces can be mirrored perfectly, i.e. the shape of the one face half reflected on the midsagittal matches the shape of the other. In reality, some form of asymmetry can be found in almost all biological structures (Lele \& Richtsmeier, 2001; Palmer \& Strobeck, 1986).

Depending on the species and populations, different forms of asymmetry are typically distinguished. In contrast to directional asymmetry (a distribution with an asymmetric average, e.g., in flatfish like flunders) and antisymmetry (bimodal distribution), fluctuating asymmetry refers to normally distributed random deviations from bilateral symmetry which are caused by both genetic and environmental perturbations. Across the population, these deviations average to a perfectly symmetric shape, i.e. a distribution with zero mean (Lele \& Richtsmeier, 2001). In psychology, fluctuating asymmetry is usually the form which is of interest when facial asymmetry is investigated.

One way to assess fluctuating facial asymmetry is the estimation of its the degree from digital frontal photographs. The shape of the face is usually digitized by annotating a set of landmarks located 
around the primary facial structures and facial outline (Farkas \& Cheung, 1981). Asymmetry scores are then calculated from the coordinates of these landmarks. In a symmetric face, for instance, the distances between landmarks and midsagittal in each face half are equal such that their total absolute differences become zero (e.g., from the nasal root to each eye; Grammer and Thornhill 1994). Contrary, the total score increases with facial asymmetry. Other scores are based on distance ratios or angles between ideally parallel lines through pairs of landmarks. The comparison of the various scores, however, is challenging since each measures a specific asymmetry feature and differs in sensitivity, magnitude, and unit (Banks et al., 2010).

The methodology by which landmarks on facial photographs are annotated also impacts asymmetry scores. In contrast to the high standardization in anthropometry and medicine (e.g. Hooda \& D’Souza, 2012), psychological studies usually require ease-of-use and practicability when gathering photogrammetric data for large scale studies. A semi-standardized approach towards head pose (position and rotation) or camera parameters (perspective or focal length) affects the coordinates of annotated landmarks and might introduce bias into asymmetry scores. For instance, if a perfectly symmetric head rotates left or right (i.e., varies yaw relative to a fixed camera), landmark distances will differ between the face halves in the photograph. Consequently, the total score of absolute differences increases with yaw independently from the actual asymmetry in the face. Such correlations have implications for the validity of the reported results.

In this respect, intrinsic and extrinsic facial asymmetry can be distinguished (Liu et al., 2003). Intrinsic asymmetry is the true asymmetry in an individual's facial shape, while extrinsic asymmetry is any acquisition-dependent factors (e.g., head poses) that affect asymmetry scores. Although methods exist to compensate for extrinsic asymmetry in facial photographs, such as variability of location and in-plane rotation (head roll) (e.g., Fink et al., 2001), head tilt and yaw usually remain in the digitized landmark coordinates as potential biases. Consequently, the validity of a scoring method depends on its ability to distinguish intrinsic from extrinsic asymmetry in facial photographs. 
To prevent biases due to extrinsic asymmetry, a possible solution would be to use 3D digital facial morphometry to score facial asymmetry from spatial face scans. Methods exist which can establish facial landmarks and filter-out pose variations from such scans completely (e.g., Claes et al., 2011; Hammond et al., 2004, White et al. 2019). It has also been demonstrated that landmarks can be established accurately in fully automatically fashion (Grewe \& Zachow, 2016, Grewe et al., 2018). 3D scanning is, however, still rarely used in psychology and photography remains the preferred data acquisition method for various reasons including affordability and ease-of-use.

\subsection{Present study}

In this work, we take advantage of 3D digital facial morphometry to investigate the relationship of head pose as a central factor of extrinsic facial asymmetry and facial asymmetry scored from facial photographs.

Major modes of facial asymmetry are empirically determined from a database of threedimensional (3D) face scans. These are then combined with major modes of symmetric facial shape variation into a 3D morphable face model. With this model, we virtually simulate a large sample of photographs with random but known variations in head pose as well as symmetric and asymmetry facial shape. Because direction and magnitude of the asymmetric shape variation is precisely determined, the simulation sample can be used for the evaluation of different facial asymmetry scoring methods.

\subsubsection{Research aim 1: evaluation of traditional methods}

Firstly, our evaluation focuses on traditional methods for the scoring of facial asymmetry (Palmer \& Strobeck, 1986). Such methods use annotated landmarks for the geometric construction of lines to measure distances and angles between facial features. We focus on two traditional morphometric methods frequently applied in either medical research (primarily oral and maxillofacial surgery), anthropology, and/or psychology. These are 1) the Horizontal Angular Asymmetry (HAA), based on 
angles (Song et al., 2007), and 2) the Horizontal Fluctuating Asymmetry (HFA), based on midpoints of horizontal lines between facial landmarks (referenced as overall facial asymmetry in Grammer \& Thornhill, 1994). Although they are used in similar ways, the actual implementation of HFA varies between published works. A major difference exists in the compensation of facial pose. We systematically investigate the correlation of these scores with intrinsic and extrinsic factors of facial asymmetry as part of study 1 . By design, we expect HFA to be severely biased by extrinsic asymmetry. HAA expectably captures a certain type of facial asymmetry only (mouth to eye).

\subsubsection{Research aim 2: establish a standardized geometric morphometrics method}

Considering the lack of standardization of traditional approaches, our second aim is to develop a common theoretically and empirically justified scoring method for facial asymmetry. We additionally want this method to resemble the principles of human face perception, which is a holistic approach. In this respect, we applied geometric morphometrics, in which the variability of all facial landmarks is simultaneously and wholly considered (Dryden \& Mardia, 1998). The method originates from the fields of evolutionary biology and anthropology (Richtsmeier et al., 2002), but has also been applied in psychological research. For instance, geometric morphometrics was used to determine the basic mechanisms of social perception from facial shape (Mayer et al., 2017).

Klingenberg and colleagues (2002) proposed an extension of geometric morphometrics for the decomposition of shape into symmetric and asymmetric components. It has been applied to determine the relationship between asymmetry of human bodies and faces with ratings of attractiveness, masculinity and femininity and other personal characteristics (Schaefer et al., 2006, van Dongen, 2014; Windhager et al., 2011).

We statistically establish a scoring method using geometric morphometrics for different types of facial asymmetry, called the Holistic Facial Asymmetry Score (HFAS), which applies to facial photographs. We demonstrate the potential of HFAS to score intrinsic and extrinsic asymmetry and its ability to distinguish between various types of facial asymmetry. 


\subsubsection{Research aim 3: evaluate the relationship between asymmetry and trait ratings}

In Study 2, we compare the different asymmetry scores on a second data set of real facial photographs. We examine relations with human ratings of the faces on their attractiveness, femininity, masculinity, and other personal characteristics. By comparing our results with previous work, we discuss the validity of HFAS and traditional methods and the potential influence of extrinsic facial asymmetry.

\section{Study 1 - evaluation of methods for the measurement of fluctuating asymmetry}

In a first step, we determined different types of facial asymmetry using 3D digital facial morphometry with a pre-existing 3D face database (Yin et al, 2006). The major symmetric and asymmetric components of facial shape variation were quantitatively assessed using principal component analysis (PCA). They were then used to build a generative 3D morphable face model. By manipulating the parameters of this model, we synthesized a random set of $N=1,000$ new and plausible 3D face shapes with varying degrees of asymmetry. With those face shapes, we created $2 \mathrm{D}$ facial photographs by virtual simulation of a typical photographic setup. With these simulated data, we established the HFAS and evaluated the scores concerning their ability to quantify intrinsic and extrinsic asymmetry.

\subsection{Study 1: methodology}

\subsubsection{Construction of a 3D morphable face model}

We used the neutral 3D surface facial scans from the BU3DFE database for $N=100$ individuals (Yin et al., 2006). The database contains variation with respect to sex ( $N=55$ females) and ethnicity $(n$ $=8$ African, $n=26$, Asian, $n=6$ Indian, $n=8$ Latino-Hispanic, and $n=51$ Caucasian face models). While there is no published information on the participants' ages, a visual inspection suggests that the age range is broad. We excluded one individual because parts of their face were covered by a scarf. For all statistical analyses, R version 3.5.0 (R Core Team, 2018) was used, along with the packages geomorph (Adams et al., 2018) and Morpho (Schlager, 2017).

To prepare the 3D scan data for a statistical shape analysis, dense face matching was performed 
utilizing a framework developed by Grewe and colleagues (Grewe \& Zachow, 2016; Grewe et al., 2018). Shape variation was decomposed into symmetric and asymmetric components using the method described by Klingenberg and colleagues (2002). By mirroring and averaging the pairwise coordinates of the two face halves (e.g., outer corner of both eyes), perfectly symmetric faces were obtained. Accordingly, the asymmetric components were found in the shape residuals. All shapes were aligned to a common average using general Procrustes analysis. We estimated Principal Components (PCs) of symmetric and asymmetric shape variation. PCs were sorted by the amount of variance explained, such that the importance of shape variation explained by the PCs decreases successively. Figure 1 displays the symmetric average face in addition to illustrations of the shape variation described by the first four asymmetric PCs. Each PC accounts for a different type of facial asymmetry. For example, the first PC captures asymmetric variation for the nose, eyes, and eyebrows, while the second includes asymmetric variation of the midface and the jaw causing a strongly bent midsagittal. 

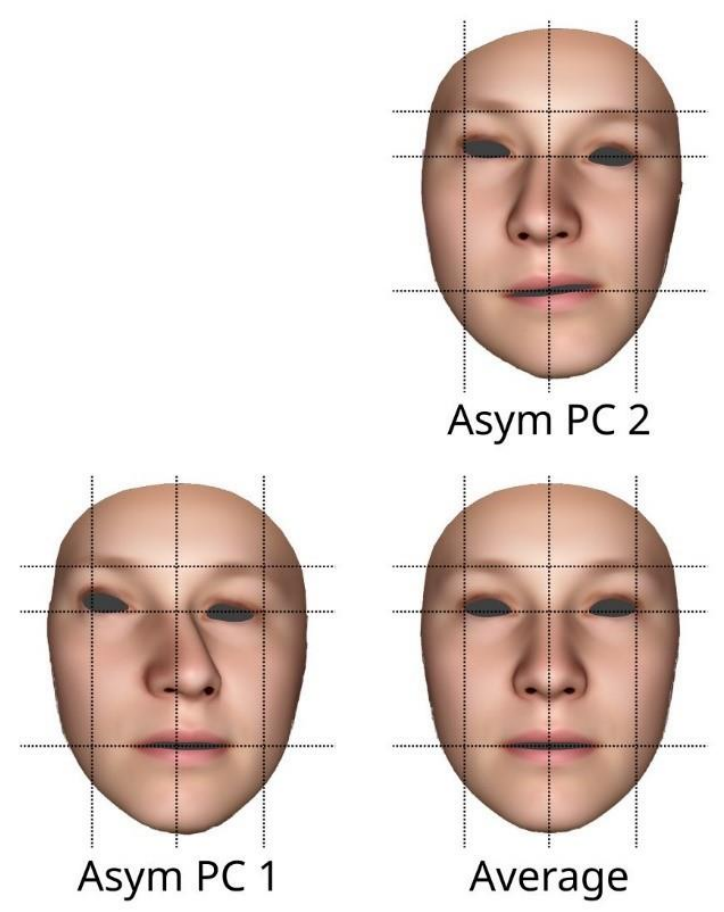

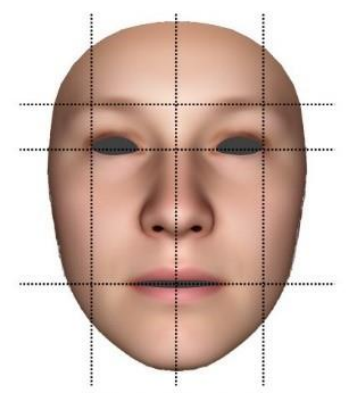

Average

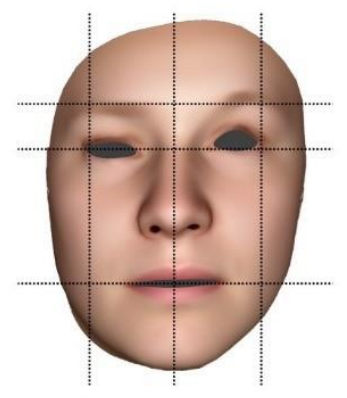

Asym PC 4

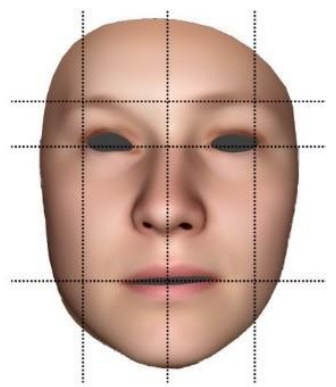

Asym PC 3

Figure 1. The symmetric average face (center) and faces showing the four most important asymmetric PCs of the 3D morphable face model. The PCs have been amplified by 8 standard deviations to illustrate the subtle asymmetries.

Next, we assembled a 3D morphable face model from the first 25 symmetric (accounting for $90 \%$ of the total variance) and four asymmetric (accounting for $50 \%$ of total variance) PCs. We set a lower cut-off for the asymmetric PCs because the signal-to-noise ratio for asymmetric shape variation is comparably worse in our database. For all PCs, a Shapiro-Wilk test (Royston, 1995) indicated normal distribution of the PC scores $(p<.05)$. Thus, the assumption of a multivariate normal distribution of the morphable face model parameters was plausible (see Figure 2). This finding indicates that the 
parameters indeed account for fluctuating asymmetry.
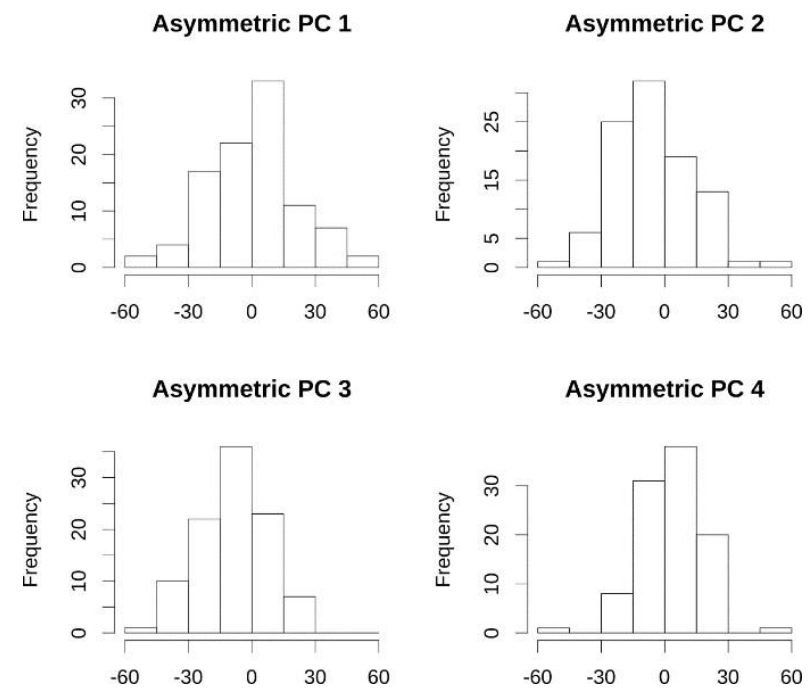

Figure 2. Histograms of the first four asymmetry PCs for the faces used to build the morphable face model.

\subsubsection{Simulation of facial photographs}

The morphable face model allows for the generation of new 3D face shapes by randomly sampling from the estimated distributions. Each generated face contains shape variation according to the 29 PCs modeled through the morphable face model (see Figure 3). $N=1000$ 3D faces were randomly drawn and rendered as facial photographs. A photographic setup for facial portraits was simulated using a virtual camera ( $85 \mathrm{~mm}$ focal length) directed towards the frontal morphable face model from a distance of $1 \mathrm{~m}$. To simulate factors of extrinsic asymmetry, three angles of head rotation (roll, pitch, and yaw) and three translations (x: posterior-anterior, y: right-left, z: superior-inferior) were randomly determined for the rendering of each facial photograph. Angles and translations were randomly sampled from a normal distribution with a standard deviation of $5^{\circ}$ and $2 \mathrm{~cm}$, respectively. These parameters were chosen to reflect the typical variability of data acquisition in the context of psychological research. The position, orientation, and internal parameters of the camera were kept constant. The software Blender (version 2.79b) and an average photographic texture were used for 
rendering. For subsequent analysis, a predefined set of $n=77$ facial landmarks (see Milborrow \& Nicolls, 2014) was determined for each simulated photograph by a perspective projection of the corresponding vertices of the morphable face model (see Figure 3).
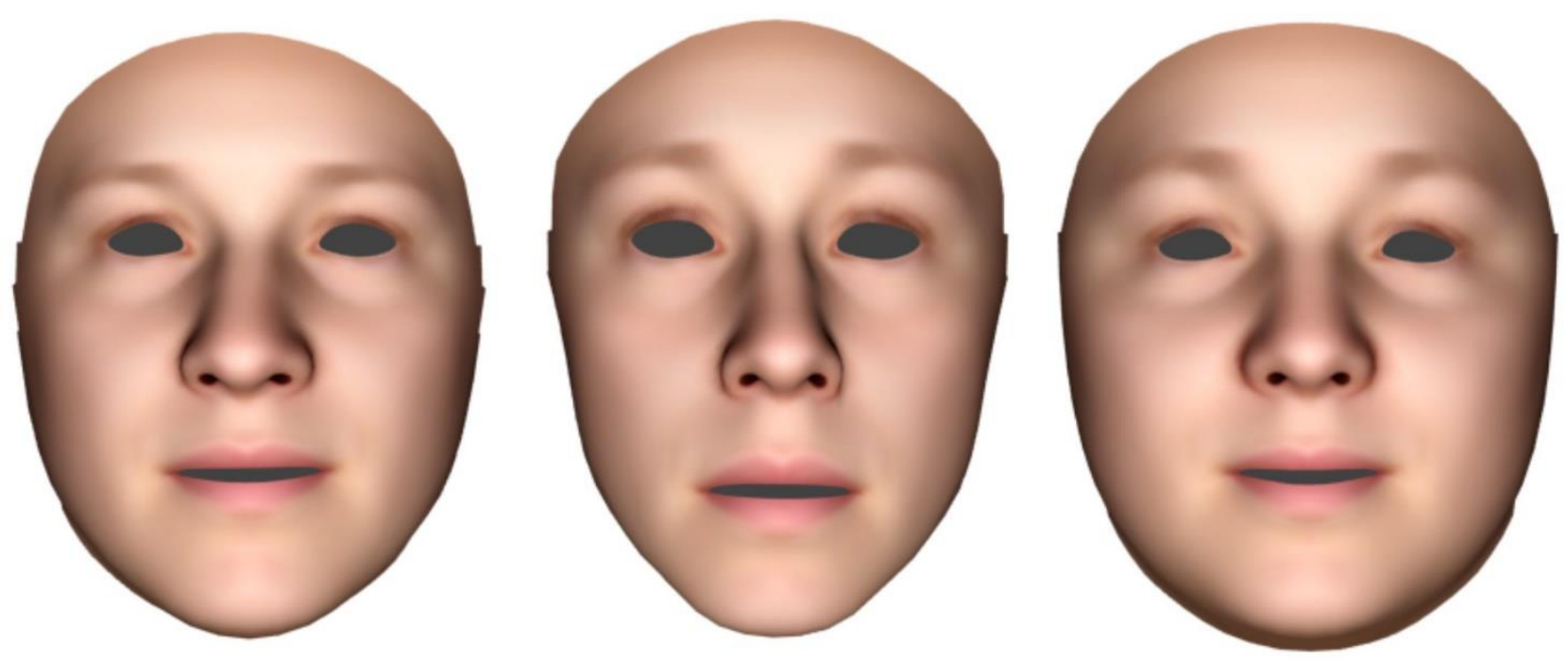

Figure 3. Examples of simulated random faces used in the present study. An average texture was used for the rendering of all photographs.

\subsection{Facial asymmetry measures based on traditional morphometric methods}

The 1,000 simulated photographs were used to compare two traditional facial asymmetry scores HFA and HAA - that were selected based on their prevalence in the literature (c.f., Borráz-León \& Cerda-Molina, 2015; Fink et al., 2006; Gangestad \& Thornhill, 1997; Garver-Apgar et al., 2008; Hooda \& D’Souza, 2012; Hönekopp et al., 2004; Koehler et al., 2004; Palmer \& Strohbeck, 1986; Penke et al., 2009; Schmid et al., 2008; Song et al., 2007; Thornhill \& Gangestad, 2006).

\subsubsection{Horizontal angular asymmetry $(H A A)$}

Horizontal angular asymmetry was estimated using the angle measure proposed by Song and colleagues (2007). HAA is the angle between a line drawn through the external corners of the eyes (i.e., the bilateral exocanthions) and a line through the external corners of the mouth (expressed in degrees). 
Because it is an angular measure, the HAA does not require pre-processing of the photographs to compensate for head roll rotation and translation.

\subsubsection{Horizontal fluctuating asymmetry (HFA)}

Facial asymmetry was assessed based on six horizontal lines between the left and right points of the outer eye corners, inner eye corners, cheekbones, nasal wings, mouth corners, and jaw as defined in Grammer and Thornhill 1994. The midpoints ( $\mathrm{mp}$ ) of all lines were calculated as:

$$
m p=\frac{X_{L}-X_{R}}{2}+x_{R}
$$

$X_{L}$ indicates a left landmark (e.g., left eye corner) and $X_{R}$ indicates a right point in the face (e.g., right eye corner). A vertical line is then defined as the average across all midpoints. On a perfectly symmetric face, all midpoints lie on the same vertical line. The HFA is a sum score based on all absolute differences between the resulting midpoints (e.g., midpoint of the facial outline minus the midpoint of the mouth).

By design, the HFA is sensitive towards extrinsic asymmetry (e.g. head roll and yaw) so that preprocessing of the landmark coordinates is required. The literature presents a variety of methods for the standardization of head orientation (Borráz-León \& Cerda-Molina, 2015; Fink et al., 2001; Fink et al., 2006; Grammer \& Thornhill, 1994; Penke et al., 2009; Scheib et al. 1999, Simmons et al. 2004). However, such adjustments are often conducted manually by untrained persons (e.g. rotation of photographs in Photoshop until the pupils are horizontally aligned). To our knowledge, an evaluation of the different methods as well as a recommendation for standardization have not been published. Due to these reasons, we refrain from adjusting the simulated landmark coordinates in our evaluation and present the degree to which intrinsic and extrinsic asymmetry are present in HFA without any preprocessing.

\subsection{Facial asymmetry measures based on geometric morphometrics}

A central goal of this study is to develop a facial asymmetry measurement technique that reflects 
human's holistic visual perception of the entire facial shape while being rigorously derived empirically. Procrustes analysis provides a standardized alignment tool to adjust in-plane rotation (head roll) and translation in facial photographs. We rigidly aligned the 77 landmarks in all 1000 simulated facial photographs to their common average shape (Dryden \& Mardia, 1998, Milborrow \& Nicolls, 2014). The 77 landmark coordinates of the average face shape also serve as the reference for the Procrustes alignment of facial landmarks annotated in real photographs. Next, the method of Klingenberg and colleagues (2002) was applied to separate symmetric and asymmetric shape variation in the aligned landmark data. Symmetric and asymmetric variations were decomposed by PCA.

\section{Study 1: results}

\subsection{Definition of HFAS from geometric morphometrics}

Figure 4 displays a thin plate spline visualization (Claude, 2008) of the shape variation described by the first five symmetric and asymmetric PCs. As expected, the Procrustes analysis of the landmarks only removed head roll and in-plane translations. A visual inspection reveals that both the first symmetric PC and the first asymmetric PC describe shape variation for factors of extrinsic asymmetry. The first symmetric PC captures variation for the pitch of the head, while the first asymmetric PC describes the head yaw, i.e., the rotation to the left or the right side. Seemingly, the remaining symmetric and asymmetric components account for regular variations in facial shape and intrinsic facial asymmetry in particular. 

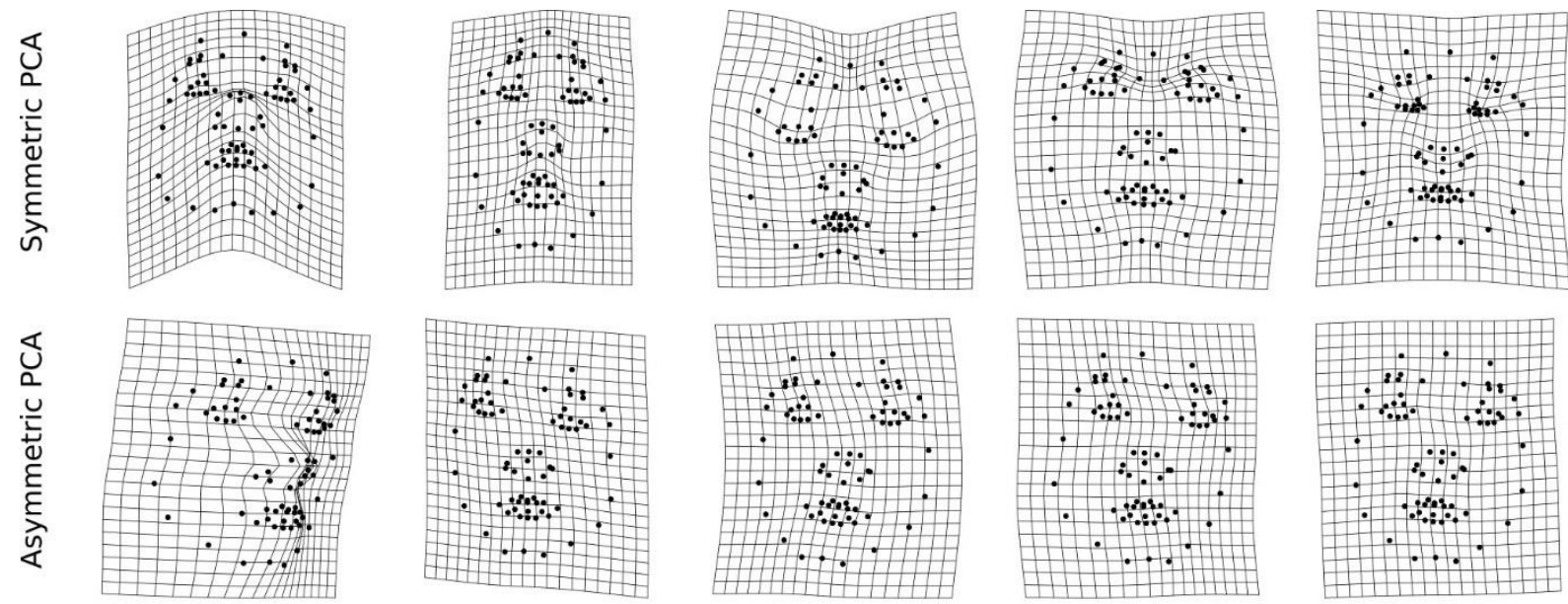

Principal components

Figure 4. First five symmetry and asymmetry PCs. Both first PCs contain out-of-plane head rotations (yaw and pitch) preserved after Procrustes analysis.

\subsubsection{Selection of the PCs}

In Figure 5, the scree plot displays the explained variance of all symmetric and asymmetric PCs. The first asymmetric PC captures about $40 \%$ of the variance, which is more than twice the amount of variance explained by the second component (15\%). This outcome can be seen as an indicator of the potential influence of head yaw on facial asymmetry measurements.
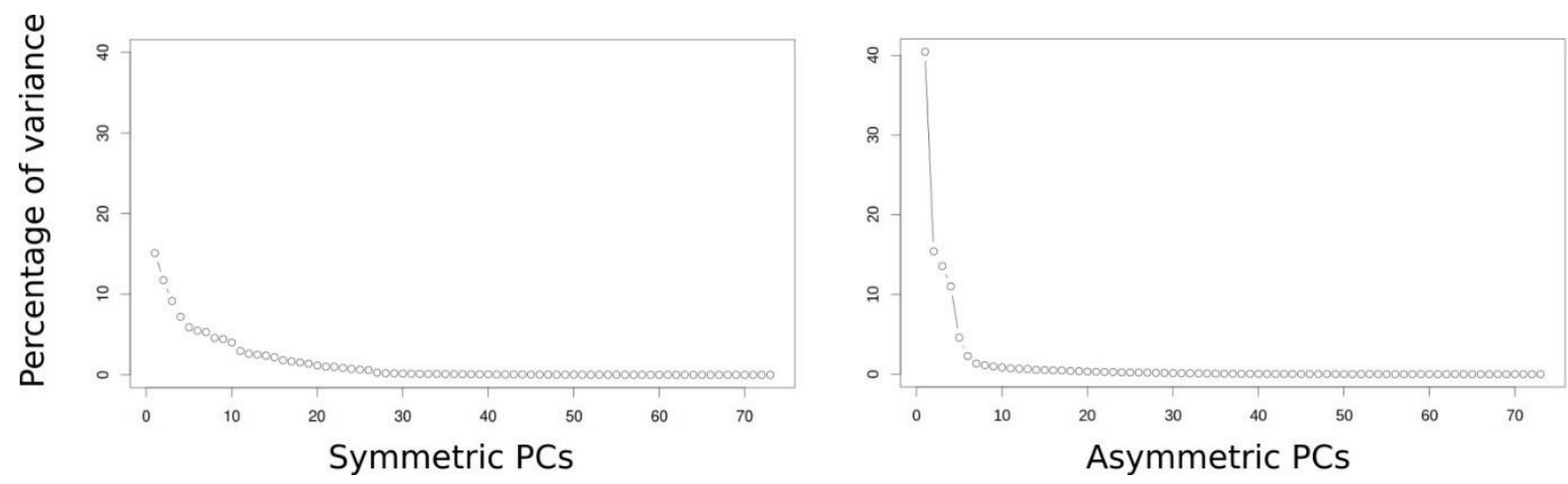

Figure 5. Scree plots for the symmetric and asymmetric PCA.

Based on the scree plot (Figure 5), we extracted five asymmetric PCs. This decision was supported by a parallel analysis (a statistical technique for determining the number of PCs; Horn, 
1965). It yielded 5 components with an adjusted Eigenvalue $>1$ (ranging from 76.75 (PC1) to 2.75 (PC5)). The number of components expectably coincides with the number of asymmetric PCs in the morphable face model plus the extrinsic factor representing head yaw.

\subsubsection{Interpretation of the PCs}

From the first symmetric and asymmetric PCs, we define HFAS-pitch and HFAS-yaw as indicators of extrinsic asymmetry. The second to fifth asymmetric PCs are interpreted as scores for different types of intrinsic facial asymmetry. From the thin plate spline visualizations (Figure 4), these PCs can be interpreted as follows: a) the second asymmetric PC displays asymmetric deviation between the left and right face halves in a superior-inferior direction (labeled as HFAS-1), b) the third asymmetric PC captures variation in the angle between the upper and lower face halves (labeled as HFAS-2), c) the fourth asymmetric PC is a combination of a lateral shift between the upper and the lower face halves as well as a superior-inferior shift between the eyes (labeled as HFAS-3), and d) the fifth asymmetry PC displays an asymmetry between the nose and the mouth (labeled as HFAS-4). Although all HFAS scores allow for determination of the direction of asymmetry, we used the absolute values in the remainder of this work to remain comparable with previous research. Also, we aggregated the four intrinsic facial asymmetry scores (PC2, PC3, PC4, and PC5) into a single measure named HFAS-total to explore the potential of quantifying asymmetry with a single score. HFAS-total was scaled to unit variance.

\subsection{Evaluation of traditional morphometric methods and HFAS}

The second aim of study 1 was to evaluate the sensitivity of HAA, HFA, and HFAS for scoring intrinsic and extrinsic facial asymmetry. It was expected that HFA suffers from the influence of extrinsic asymmetry and is a less valid indicator of intrinsic asymmetry. HAA was expected to correlate with a specific type of intrinsic asymmetry while HFAS should be able to predict various types of asymmetry. Because the sample of facial photographs was simulated using the morphable face model, symmetric and asymmetric facial shape characteristics were known. Thus, we could correlate 
asymmetry scores with the absolute rotations and translations (see Table 1).

Table 1

Correlations between traditional morphometric measures (HAA and unadjusted HFA), the geometric morphometric HFAS, and known acquisition-related factors for head translation $(X, Y$, Z) and rotation (Roll, Pitch, Yaw) in Study 1.

\begin{tabular}{|c|c|c|c|c|c|c|}
\hline & \multicolumn{3}{|c|}{ Head Translation } & \multicolumn{3}{|c|}{ Head Rotation } \\
\hline & $\mathrm{X}$ & $\mathrm{Y}$ & $\mathrm{Z}$ & Roll & Pitch & Yaw \\
\hline \multicolumn{7}{|c|}{ Traditional morphometric methods } \\
\hline HAA & -0.02 & -0.03 & 0.02 & 0.00 & 0.00 & 0.05 \\
\hline HFA & $-0.07 *$ & -0.03 & 0.03 & $0.92 * * *$ & -0.01 & $0.29 * * *$ \\
\hline \multicolumn{7}{|c|}{ Geometric morphometric method } \\
\hline HFAS-yaw & $-0.08 * *$ & -0.03 & -0.00 & 0.06 & 0.01 & $0.81 * * *$ \\
\hline HFAS-pitch & 0.00 & 0.00 & 0.03 & -0.03 & $0.68 * * *$ & 0.00 \\
\hline $\begin{array}{l}\text { HFAS-1 (left-right } \\
\text { face half } \\
\text { asymmetry) }\end{array}$ & -0.01 & $0.06 *$ & 0.03 & -0.06 & $0.10 * *$ & $0.13 * * *$ \\
\hline $\begin{array}{l}\text { HFAS-2 (upper- } \\
\text { lower face half } \\
\text { asymmetry) }\end{array}$ & 0.03 & -0.04 & 0.04 & -0.05 & 0.02 & -0.02 \\
\hline $\begin{array}{r}\text { HFAS-3 (bended } \\
\text { midsagittal line) }\end{array}$ & -0.02 & 0.01 & -0.03 & $0.07 *$ & -0.02 & 0.06 \\
\hline $\begin{array}{l}\text { HFAS-4 (mouth- } \\
\text { nose asymmetry) }\end{array}$ & -0.03 & 0.02 & 0.00 & 0.03 & 0.02 & -0.01 \\
\hline HFAS-total & -0.01 & 0.03 & 0.02 & -0.03 & $0.08 *$ & $0.11 * * *$ \\
\hline
\end{tabular}

\subsubsection{Relations with extrinsic asymmetry}

As expected, HFA was significantly correlated with head roll $(r=0.92, p<.001)$, yaw $(r=0.29$, $p<.001)$, and weakly with translation in $\mathrm{x}$-direction $(r=-0.07, p<.05)$. Similarly, high correlations were found between HFAS-yaw and yaw $(r=0.81, p<.001)$, as well as between HFAS-pitch and pitch $(r=0.68, p<.001)$. To test the convergent validity of these two scores, these results were compared to 
a recent face tracking framework (Baltrusaitis et al., 2018). Tracking was performed for the simulated photographs and yielded similar scores (yaw: $r=0.77, p<.001$; pitch: $r=0.62, p<.001$ ). HFAS is thus on par with state-of-the-art methods for determining out-of-plane rotations as indicators of extrinsic facial asymmetry. Also, there were weak but significant correlations with extrinsic asymmetry and HFAS-1, HFAS-3, and HFAS-total. HAA was not related to any tested factors of extrinsic facial asymmetry.

\subsubsection{Relations with intrinsic asymmetry}

Next, estimates of intrinsic asymmetry were examined by correlating traditional asymmetry scores and HFAS with the absolute values of the simulated asymmetry types contained in the morphable face model (see Table 2). HFA was not correlated with any of the simulated asymmetries. However, HAA estimation was weakly related to the first PC of the morphable face model and strongly with its second PC. As shown in Figure 1, the first PC displays an asymmetry on the midsagittal line (shifted nose and mouths compared to the eyes) while PC 2 shows an asymmetry of nose to mouth. Therefore, the correlational patterns of HAA and PC1 and PC2 are theoretically highly meaningful, as HAA is a measure of the eye-mouth angle.

As expected, HFAS-yaw and HFAS-pitch were only weakly correlated or entirely unrelated with the known face model asymmetries. Remarkably, the deviation between left and right face halves (HFAS-1) was strongly correlated with PC1 in the morphable face model and weakly related to the last PC. The angle between the upper and lower face half (HFAS-2) showed a very strong correlation with the second PC in the morphable face model and a weak correlation with the third PC. The shift between upper and lower face half and the eyes (HFAS-3) was moderately related with all morphable face model PCs. The mouth-nose asymmetry (HFAS-4) was strongly related with the morphable face model PC3 and PC4. HFAS-total was positively related to all four morphable face model PCs. 
Table 2

Correlations between traditional morphometric measures (HAA and HFA), the geometric

morphometric HFAS, and known asymmetry parameters of the 3D morphable face model in

Study 1. The correlations of HFAS, distances, and HFA are based on absolute values.

\begin{tabular}{lcccc}
\hline & \multicolumn{4}{c}{ Principal Components $(\mathrm{PCs})$ of the morphable face model } \\
& PC1 & PC2 & PC3 & PC4 \\
\hline HAA & $\mathbf{0 . 1 6} * * *$ & $\mathbf{0 . 6 2} * * *$ & 0.05 & -0.03 \\
HFA & 0.05 & -0.04 & 0.02 & 0.02 \\
HFAS-yaw & $\mathbf{0 . 1 3} * * *$ & -0.01 & 0.02 & 0.00 \\
HFAS-pitch & 0.00 & -0.04 & 0.02 & -0.02 \\
HFAS-1 & $\mathbf{0 . 5 6 * * *}$ & 0.03 & 0.04 & $\mathbf{0 . 0 9} * *$ \\
HFAS-2 & -0.04 & $\mathbf{0 . 7 1} * * *$ & $\mathbf{0 . 1 4} * * *$ & 0.02 \\
HFAS-3 & $\mathbf{0 . 2 1} * * *$ & $\mathbf{0 . 0 9} * *$ & $\mathbf{0 . 2 0} * * *$ & $\mathbf{0 . 2 7} * * *$ \\
HFAS-4 & $\mathbf{0 . 0 3}$ & $\mathbf{0 . 0 3}$ & $\mathbf{0 . 4 7} * * *$ & $\mathbf{0 . 4 5} * * *$ \\
HFAS-total & $\mathbf{0 . 4 6 * * *}$ & $\mathbf{0 . 4 6} * * *$ & $\mathbf{0 . 2 9} * * *$ & $\mathbf{0 . 2 9} * * *$ \\
\hline
\end{tabular}

Note. Coefficients in boldface are statistically significant $(* p<.05 ; * * p<.01 ; * * * p<.001)$.

\section{Study 1: summary and discussion}

\subsection{Traditional methods}

We found that our implementation of HFA greatly suffers from an influence of extrinsic facial asymmetry (i.e., head roll and yaw). Even if head roll is compensated for, it is affected by head yaw. Moreover, HFA was not related to any of the tested asymmetry patterns. In the light of these findings, it must be stressed that HFA performs poorly as a measure of intrinsic facial asymmetry. As the literature lacks a standardized procedure for such an adjustment, its application must be deemed problematic.

This has remarkable implications for the interpretation of previous works that use HFA to score facial asymmetry.

HAA did not suffer from extrinsic facial asymmetry factors. Also, it was highly correlated with the specific facial asymmetry pattern described by the morphable face model PC2. As it measures the angle between two lines, each connecting the mouth and eye corners, this measurement is in-line with the illustration of the according shape variation shown in Figure 1. HAA was unable to indicate other types of facial asymmetry patterns which were determined from the 3D face database. 


\subsection{Holistic facial asymmetry score}

The correlation patterns substantiated that HFAS-yaw and HFAS-pitch explain a large proportion of variation in these aspects of head pose. Additionally, HFAS-1 to HFAS-4 were largely related to the types of asymmetry patterns contained in the morphable face model. By comparing the 3D shape variations illustrated in Figure 1 to their planar counterparts in Figure 4, their relationship becomes apparent. As HFAS-total is an aggregated score derived from HFAS-1 to HFAS-4, it is not displayed in the figures.

We propose HFAS as a geometric morphometric-based method that can be readily applied to real facial photographs to score various types of facial asymmetry. After annotation of the $n=77$ landmarks for a new picture, they can be aligned to the reference landmarks (i.e. the average face shape) using Procrustes analysis. By projection onto the six components of HFAS, one can then score the different types of intrinsic and extrinsic asymmetry. Because it can be largely automatized, it is also a suitable tool for the analysis of large image databases.

Our results suggest that the scores for HFAS-pitch and HFAS-yaw are indicators of noise resulting from semi-standardized picture acquisition setups. The scores represent of out-of-plane head rotations, which cannot be easily removed by relative measures or Procrustes analysis. They can be used as indicators of extrinsic facial asymmetry to either discard high-scoring images before facial asymmetry analysis or to include them as confounding covariates in a subsequent analysis.

Likewise, the separation of HFAS from extrinsic facial asymmetry allows to score various types of intrinsic facial asymmetry. HFAS-1 to HFAS-4 account for the magnitude and direction of different asymmetries that are jointly present in a facial photograph. We therefore argue that HFAS-total can serve as an aggregate measure of overall facial asymmetry.

\subsection{Limitations}

Admittedly, the quality of our simulation depends on the shape variability that is statistically extracted from the 3D face database using digital facial morphometry. Because we used a small sample 
of $N=99$ face scans, the representativity of the morphable face model might be limited and more diversity in our database is desirable. However, only the most important PCs were retained to define HFAS. These PCs usually generalize well to larger and more diverse databases; thus, we have likely identified common types of facial asymmetry. Nevertheless, HFAS can be easily extended and future research with extended 3D databases may provide information about the sample size needed to saturate the analysis of different types of facial asymmetry. Generally, we believe that HFAS has great potential as a more holistic approach to score facial asymmetry in applied work.

\section{Study 2 - application of HFAS to observed facial photographs}

In study 1 , HFAS was developed to capture facial shape using $n=77$ facial landmarks. However, because the landmark locations were simulated, the validity of the method for real photographs is unclear. With study 2, we evaluated the predictive validity of HFAS for real photographs. Specifically, HFAS components were related to trait characteristic ratings. Because HAA was the least related with specific types of facial asymmetry, its predictive validity was also evaluated. Additionally, HFAS was qualitatively assessed by frontalizing and symmetrizing facial photographs using image warping.

\subsection{Study 2: methodology}

The facial photographs were collected as part of a larger study on socio-emotional abilities (Kaltwasser et al., 2016) and analyzed for facial width in Kaltwasser and colleagues (2017). The ratings of the facial photographs have not been considered in any previous publication. Participants were asked to remain with a neutral face expression when they were photographed with a Panasonic HDC-SD707 camera. The camera was mounted on a tripod in front of a grey background at a $1.5 \mathrm{~m}$ distance from the participants, who were instructed to look directly into the camera. The color portraits of $N=186$ participants who gave consent to their picture being used for further analyses were preprocessed and cut into rectangular facial images of the same size (e.g., removing the presence of the neck and the remaining space above the head) using Photoshop. The resolution of these images was resized to approximately $330 \times 400$ pixels. 
Because manual annotation is laborious, a publicly available automatic landmark detector (Milborrow \& Nicolls, 2014) was used to determine the 77 landmarks for each photograph. The landmark annotations were then manually checked for accuracy by the first authors, with 26 photographs excluded because the detector failed to correctly identify facial structures (mostly the landmarks describing the facial outline). We found that the detector worked reliably in all other cases. The final sample consisted of $N=160$ images of Caucasian adults ( $45 \%$ female) with a mean age of 27.6 years $(S D=5.7)$. The study was conducted following the Declaration of Helsinki and approved by the ethics committee of the Department of Psychology (protocol approval number 2013-17). All participants provided written consent before the start of the experiment.

\subsection{Ratings}

Next, all participants from the Kaltwasser et al. (2016) study $(N=210)$ were contacted via email and asked to rate all 186 facial images. Ratings were made on the online platform Unipark (@) QuestBack $\mathrm{GmbH})$. From the original sample, 81 persons (51\% female; $M=27.7$ years, $S D=5.7$ ) participated.

Participants were asked to rate each face on facial distinctiveness, attractiveness, femininity/masculinity, and intelligence. Additionally, since the ratings were collected within a study investigating individual differences in fairness processing, participants were asked to rate the faces on antisocial behavior (assertiveness), fairness, greed, modesty, negativity, sincerity, and the supposed frequency of social interaction with others (sociability). Participants were provided with a definition of each rating dimension, so they were clear on their meaning. The list of traits was presented below the facial image with a 7-point Likert scale response format (ranging from not at all to very much). A randomly-generated order of the traits was fixed across facial images and participants, and the order of the facial images was reversed for half of the rating sample. The ratings were $z$-standardized and aggregated across all raters to per-individual scores. The anonymized rating data is available in the Open Science Framework https://osf.io/f34h7/. 


\subsection{Application of geometric morphometrics and computation of the HFAS}

The average reference face and symmetric/asymmetric PCA models determined in study 1 were used to compute the HFAS for the landmarks annotated in the real facial photographs. To this end, a fitting algorithm was applied to each landmark configuration which alternates between (1) Procrustes alignment of the landmarks and (2) least-squares projection of the aligned landmarks onto the symmetric and asymmetric PCs determined in study 1 . The alternating optimization scheme was repeated and typically converges in 5 to 8 iterations reaching the tolerance threshold of $1 \mathrm{e}-12$. The HFAS for intrinsic and extrinsic facial asymmetry were determined from the least squares projection onto the respective PCs.

To qualitatively assess the intrinsic and extrinsic facial asymmetry patterns described by the HFAS, the corresponding facial patterns can be manipulated in the original photographs. The shape of the individual landmarks can be altered by adjustment of the HFAS values. The same shape change can be applied to the facial photographs using thin plate spline image warping.

Using the warping technique, we aim at testing HFAS ability to score extrinsic facial asymmetry. We frontalized versions of high-scoring facial photographs on HFAS-pitch and HFAS-yaw by setting the corresponding individual scores to zero and warping the images according to the changes in face shape. Similarly, cases with low and high intrinsic facial asymmetry were identified from a ranking of the photographs by HFAS-total. By removing the HFAS asymmetries from the landmarks, symmetrized photographs were synthesized. In both cases, the face image was cut along the outline provided by the landmark configuration.

\section{Results of study 2}

Examples of original and frontalized photographs after HFAS-pitch and HFAS-yaw adjustment are displayed in Figure 6. The extrinsic asymmetry factors which are not adjusted through Procrustes analysis (i.e., head pitch and yaw) were successfully identified by HFAS-yaw and HFAS-pitch and could partly be removed by image warping towards a frontal picture. 

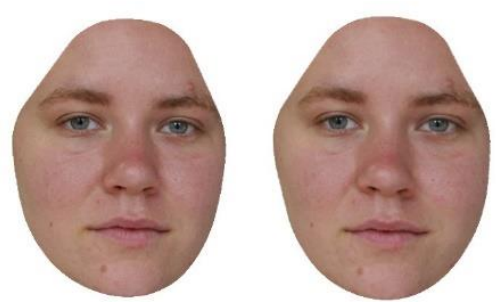

Original Frontalized
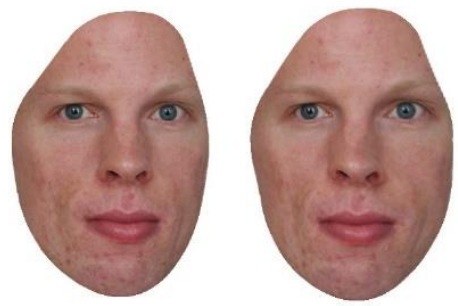

Original
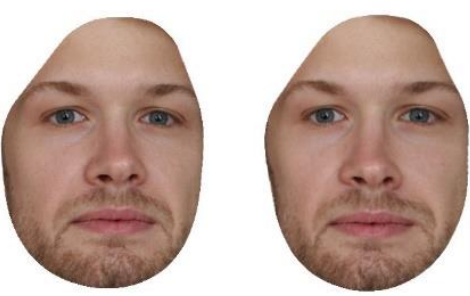

Original

Frontalized

Figure 6. HFAS-based frontalization of facial photographs. The original version is shown along with the face after removal of head pitch and yaw.

Figure 7 illustrates faces with low and high intrinsic facial asymmetry scores on HFAS. Along with the original photographs, manipulated faces with amplified facial asymmetry (HFAS-1 to HFAS-4 is doubled in magnitude) as well as the symmetrized versions (zero HFAS-1 to HFAS-4) are depicted. All faces have been frontalized in advance according to their scores on HFAS-yaw and HFAS-pitch to avoid visual disturbance. As illustrated, HFAS-total correctly identifies asymmetric and symmetric faces. 


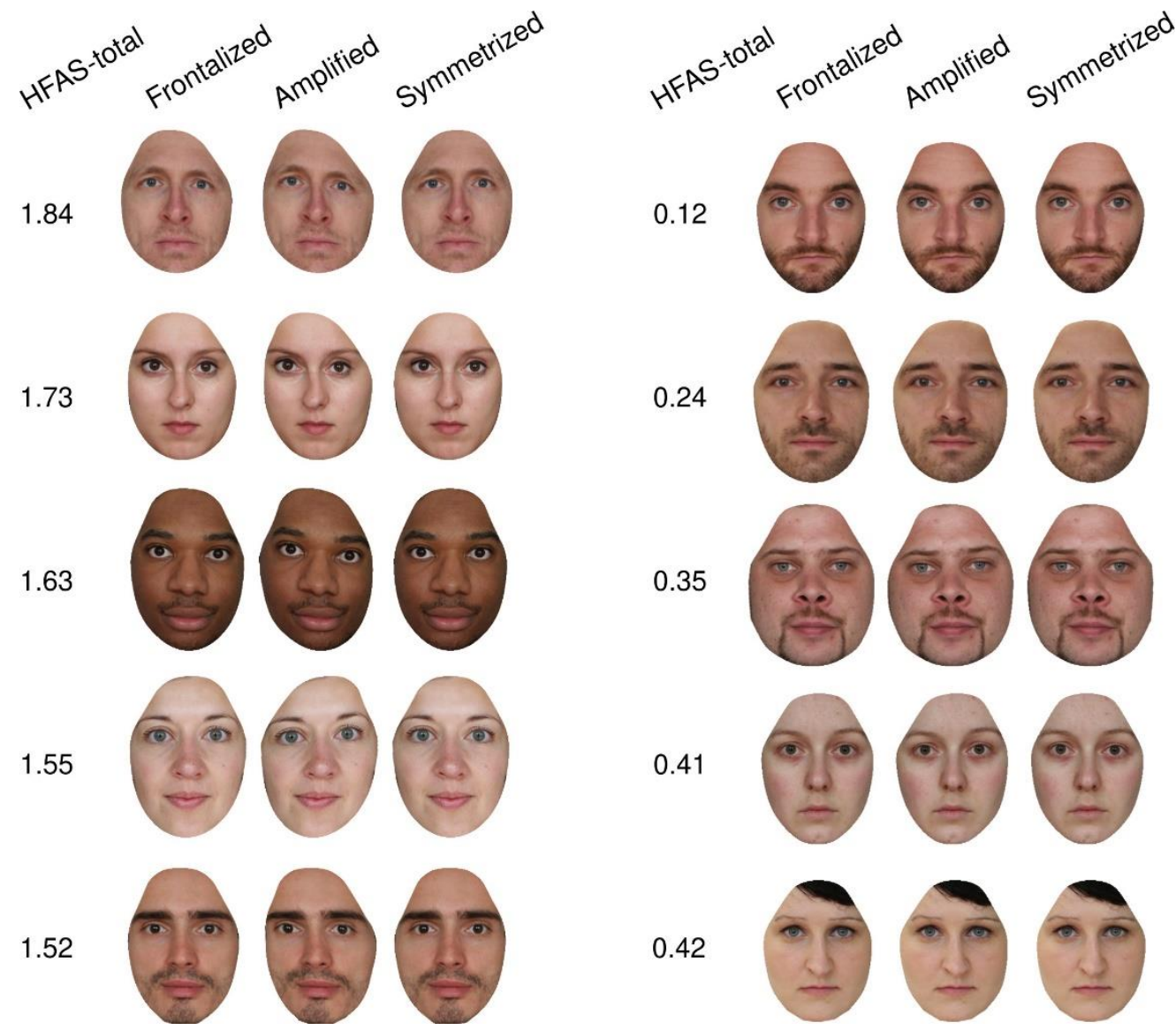

Figure 7. HFAS-based scoring and symmetrization of observed facial photographs: Faces with high HFAS-total (left column) and low HFAS-total (right column). Symmetric faces were synthesized (Symmetrized) by adjusting the shape in the frontalized photographs (Frontalized). Because asymmetry patterns in regular faces are often subtle, they have been amplified by a factor of 2 for visualization (Amplified).

Lastly, the predictive validity of HFAS and HAA was evaluated. This was done by testing correlations between the facial asymmetry estimates with ratings of facial attractiveness and the other traits (see Table 3). Except for HFAS-pitch, where negative/positive HFAS-pitch indicates a bowed/lifted head pose respectively, absolute HFAS scores were used. As will become obvious below, the direction of head pitch is important. For all intrinsic asymmetry measures, a negative correlation does not consequently implicate the direction of asymmetry but rather the degree of asymmetry. For 
example, the negative relation between HAA with masculinity indicates that less asymmetry is perceived as more masculine. According to the HAA, more asymmetry was rated as more fair, feminine, modest, and sincere, while less asymmetry was rated as greedier and more masculine. HFASyaw was unrelated to all of the collected ratings. The correlation of HFAS-pitch indicates that the extrinsic head pitch is related with attractiveness, i.e. bowed faces are rated more attractive and feminine. In contrast, a lifted face is perceived as more negative and masculine.

The asymmetry of the left and right face halves (HFAS-1, defined in Study 1) was not significantly related with any of the rated traits. More asymmetry between the upper and the lower face halves (HFAS-2) meant the person was perceived as less feminine, more masculine, and greedy. More asymmetry along the bent midsagittal line (HFAS-3) was also not related to any of the rated traits. A greater asymmetry between the nose and the mouth (HFAS-4) was perceived as being less negative, more fair, and sincere. Finally, the overall asymmetry score HFAS-total was not significantly associated with the attributed traits. 
Table 3

Observed Data Study 2: HAA, HFAS-components (including extrinsic asymmetry: yaw and pitch) with rating data.

\begin{tabular}{|c|c|c|c|c|c|c|c|c|c|c|}
\hline & Antisocialness & Attractiveness & Negativity & $\begin{array}{c}\text { Facially } \\
\text { distinctiveness }\end{array}$ & Fairness & Femininity & Greediness & Masculinity & Modesty & Sincerity \\
\hline HAA & -0.11 & 0.05 & -0.10 & 0.10 & $0.17 *$ & $0.22 * *$ & $-0.17 *$ & $-0.22 * *$ & 0.18* & 0.18* \\
\hline $\begin{array}{r}\text { HFAS- } \\
\text { yaw }\end{array}$ & -0.05 & -0.05 & 0.01 & 0.02 & 0.09 & -0.08 & -0.01 & 0.06 & 0.09 & 0.06 \\
\hline $\begin{array}{l}\text { HFAS- } \\
\text { pitch }\end{array}$ & 0.06 & $-0.26 * * *$ & $0.16 *$ & 0.09 & -0.13 & $-0.25 * *$ & 0.12 & $0.24^{* *}$ & -0.13 & -0.11 \\
\hline $\begin{array}{l}\text { HFAS- } \\
1\end{array}$ & -0.13 & -0.04 & -0.02 & -0.05 & 0.08 & -0.01 & -0.07 & -0.01 & 0.15 & 0.07 \\
\hline $\begin{array}{l}\text { HFAS- } \\
\quad 2\end{array}$ & 0.10 & -0.08 & 0.09 & 0.05 & -0.14 & $-0.19 *$ & $0.17 *$ & $0.18 *$ & -0.13 & -0.13 \\
\hline $\begin{array}{l}\text { HFAS- } \\
\quad 3\end{array}$ & -0.04 & -0.04 & -0.03 & 0.07 & 0.01 & 0.04 & -0.03 & -0.06 & 0.03 & 0.04 \\
\hline $\begin{array}{c}\text { HFAS- } \\
4\end{array}$ & -0.01 & 0.14 & $-0.19 *$ & 0.03 & $0.22 * *$ & 0.05 & -0.09 & -0.04 & 0.10 & $0.20 * *$ \\
\hline $\begin{array}{l}\text { HFAS- } \\
\text { total }\end{array}$ & -0.07 & -0.03 & -0.04 & 0.02 & 0.07 & -0.05 & -0.02 & 0.03 & 0.09 & 0.07 \\
\hline
\end{tabular}

Note. Coefficients in boldface are statistically significant $* p<.05 ; * * p<.01 ; * * * p<.001$ 


\section{Study 2: summary and discussion}

Overall, the applicability of HFAS for scoring extrinsic and intrinsic facial asymmetry for real photographs was qualitatively demonstrated. In principle, the identification of extrinsic facial asymmetry, together with the image warping technique, provides a way to remove extrinsic facial asymmetry from photographs. For prospective rating studies, this ability could be especially useful when pictures were acquired in semi-standardized settings. If the removal of extrinsic factors from photographs is infeasible, the extrinsic HFAS scores may serve as indicators to control potential confounds.

We also evaluated the predictive validity of HAA and HFAS by testing bivariate relationships between intrinsic facial asymmetry scores with the trait ratings. We chose to evaluate only HAA and HFAS because they were less affected by extrinsic asymmetry and at least correlated with one of the simulated types of asymmetry in study 1. Even though most previous results in the psychological literature were obtained using HFA, it was omitted from further analysis. Our reasons were that (a) it was not related to any of the observed asymmetry types in our implementation and (b) its implementation differs across previous studies such that the evaluation of its predictive validity becomes particularly challenging (Banks et al., 2010).

\subsection{Perceived attractiveness}

The positive relationship between facial asymmetry and attractiveness has been discussed controversially (e.g., Weeden \& Sabini, 2005, Grammer et al. 2005). For example, the research of Grammer and Thornhill (1994), Scheib and colleagues (1999), and Fink and colleagues (2006), all of which use different variants of the HFA, reported that stronger symmetry is associated with higher ratings of attractiveness. However, in their review, Weeden and Sabini (2005) found that correlations are heterogeneous and often not significant. Similarly, van Dongen (2014) found no correlation between facial symmetry and attractiveness ratings using geometric morphometrics to determine asymmetry scores. Our results also support the conclusion of a null correlation. Moreover, the 
association of attractiveness ratings and HFAS-pitch found in study 2 is in line with the findings of Sulikowski et al. (2015). These findings provide a first hint on the predictive validity of HFAS.

\subsection{Perceived femininity/masculinity}

Additionally, our correlational results show that intrinsic asymmetry scores are related with ratings of femininity and masculinity. Specifically, the type of facial asymmetry which is scored by HFAS-2 is positively and negatively correlated with ratings of masculinity and femininity respectively. Previous research from evolutionary biology and psychology investigated the relationship between facial asymmetry as markers of developmental instability and (biological) sexual dimorphism. In the majority of the studies, traditional scores such as HFA were used but the achieved results were heterogeneous. The meta-analysis of van Dongen (2012) reported an average effect size of nearly zero. A study using facial asymmetry scores captured by geometric morphometrics also found no association with sexual dimorphism (van Dongen 2014). On the contrary, Koehler (2004) found a negative association between facial and body asymmetry with rated femininity using traditional scores (the relationship of facial asymmetry was rather unstable than for body asymmetry). Mitteroecker and colleagues (2015) reported a positive correlation of geometric morphometric-based asymmetry scores with ratings of masculinity. These findings provide additional support for the validity of HFAS.

\subsection{Perceived personality}

Our results revealed an agreement in correlational patterns between HAA and HFAS-4 for some personality ratings. Higher facial asymmetry scores were related with less negativity, greater fairness, and greater sincerity. Similar to HFAS-2, HAA was also correlated with ratings of femininity and masculinity but the correlations were opposite in their sign. The reason might be that HAA scores a single aspect of intrinsic facial asymmetry, which might be inadequate with femininity/masculinity ratings due to their dependence on various features across the face (Koehler et al., 2004). A more holistic approach like HFAS, as well as its sensitivity to distinguish between different types of intrinsic facial asymmetry arguably, allows one to draw a more appropriate and detailed picture for studying 
relationships with human perceptions. Nevertheless, a further investigation of the differences between HAA and HFAS is required.

\subsection{Evaluation of the HFAS-total}

Interestingly, the HFAS-total was not significantly associated with any of the rating dimensions. This suggests that the ad-hoc definition of aggregate scores, such as in HFA, might be insufficient to investigate the relationships between facial asymmetry and trait ratings. HFAS $1-4$ were on the other hand statistically learned by PCA decomposition of asymmetric shape variation from a database of real face scans. They thus resemble the morphometric distribution of major types of facial asymmetry. Arguably, such a data-driven definition is more compatible with the features of mental face representation in humans.

\section{Overall conclusion and future directions}

The presented work aimed at evaluating traditional methods for the scoring of facial asymmetry from photographs. We have distinguished two potential sources of variability in such scores: intrinsic and extrinsic facial asymmetry. Both kinds were specifically manipulated in a simulated sample of virtual photographs based on face synthesis from a 3D morphable face model. Our evaluation of two traditional asymmetry scores showed mixed results. The HAA scores a specific type of intrinsic asymmetry, i.e. the angle between two lines through the eye and mouth corners, and was not related to extrinsic factors. The widely applied, distance-based HFA score did not show any correlation to intrinsic but was substantially affected by extrinsic facial asymmetry, namely head roll and yaw. Due to the diversity of implementations in previous works, we evaluated HFA without compensation of inplane orientation. Although further analysis of the different HFA variants is needed, preliminary results show a consistent picture with the results reported above. Random or systematic variation in factors of extrinsic asymmetry occurs easily when faces are captured with semi-standardized photogrammetric setups. Consequently, scoring methods are needed which provide a detailed view of different factors of intrinsic facial asymmetry and are, at the same time, robust against extrinsic factors. 
To account for the shortcomings of traditional facial asymmetry scores, a novel method called Holistic Facial Asymmetry Score (HFAS) was developed using a combination of digital 3D facial morphometry and geometric morphometrics. Although it is particularly challenging to separate extrinsic and intrinsic asymmetry in facial photographs, we have demonstrated the benefits of HFASpitch and HFAS-yaw as reliable indicators of extrinsic facial asymmetry. In combination with computer graphics techniques, HFAS might further be used to control certain features of intrinsic and extrinsic asymmetry by warping photographs towards more symmetric faces.

By statistically learning types of intrinsic facial asymmetry, HFAS-1 to HFAS-4 were established without the need for ad-hoc formalisms and definitions, which are often the basis of traditional methods. To our knowledge, this study is the first to provide a rigorously data-driven method for scoring facial asymmetry from photographs. Although further research is needed, such a method that resembles the morphometric distribution of real faces is arguably more compatible with holistic face processing and perception in humans. It is thus particularly suited for the investigation of the relationship between facial asymmetry and ratings.

A convenient application of our method to new frontal facial photographs is facilitated by an automatic scoring method to compute HFAS. To clarify the effect of automatic landmark detection, we will further examine the reliability and robustness of such algorithms and potential biases in HFAS scores. But also without automatic landmark detection, we hope to contribute towards better standardization and interpretation of prospective research on facial asymmetry.

Our future research will be directed towards advancements in 3D facial morphometry that facilitate convenient analysis of 3D face scans. This will not only allow psychological researchers to use scores that are robust against factors of extrinsic facial asymmetry present in conventional photogrammetry. It also helps us to enlarge our morphable face model to discover additional components of intrinsic asymmetry for the extension of HFAS. In addition to a sparse set of landmarks determined from facial photographs, the analysis of facial asymmetry will also be performed densely 
by capturing the entire facial shape with arbitrarily high resolution (Grewe and Zachow, 2016, Grewe et al. 2018).

In conclusion, we believe HFAS might help future research clarify the role of facial asymmetry in the perception of faces, such as sexual dimorphism and the attribution of gender stereotypes. Other research directions that were not mentioned in this paper include the contribution of facial asymmetry in the field of facial expressions of emotion such as the ability to perceive (Hildebrandt et al., 2015) and express emotions (Olderbak et al., 2014). Likewise, it is important to further investigate the relationship of facial asymmetry and clinical disorders, also covering neurological research and facial asymmetry. Last but not least, HFAS might be usefully applied in medical contexts, for instance, to compare asymmetry pre- and postoperatively in facial plastic surgery (Ko et al., 2009, Zachow 2015).

\section{Acknowledgments}

This research was supported by the Deutsche Forschungsgemeinschaft with grants awarded to Andrea Hildebrandt and Werner Sommer (HI1780/2-1 and SO 177/26-1). C. Martin Grewe was funded by the Image Knowledge Gestaltung Cluster of Excellence at the Humboldt-Universität zu Berlin, as a part of the Excellence Initiative of the German Research Foundation (Grant no. EXC 1027). The funders had no role in study design, data collection and analysis, decision to publish, or preparation of the manuscript. We thank Oliver Wilhelm for his helpful advice throughout the project. We thank Danyal Ansari, Tsvetina Dimitrova, Lena Fliedner, Nina Mader, Katariina Mankinen, Kerry Moore, Friederike Rüffer, and Susanne Stoll for their help in recruitment and data collection. The facial photographs were collected as part of a larger study on socio-emotional abilities (Kaltwasser, Hildebrandt, Wilhelm, \& Sommer, 2016) and analyzed with respect to facial width in Kaltwasser, Mikac, Busko, and Hildebrandt (2017). For all studies, we have reported conditions and data exclusions. Decisions surrounding final sample sizes are presented in the sample sections.

\section{Availability of scripts and data}

R-scripts used for the analysis of the simulated and observed faces are available in the Open Science 
Framework https://osf.io/f34h7/. We are also planning to release a software package that allows for the convenient determination of HFAS in new photographs. We hope that our scoring procedure will be helpful for researchers and practitioners studying facial asymmetry.

\section{Credit Author Statement}

Selina Weiss: Conceptualization, Methodology, Formal Analysis, Writing - Original Draft, Writing review \& editing, Validation, Data Curation, Visualization. C. Martin Grewe: Conceptualization, Methodology, Formal Analysis, Writing - Original Draft, Writing - review \& editing, Software, Data Curation, Visualization. Sally Olderbak: Conceptualization, Writing - Original Draft, Writing Review \& Editing. Benjamin Goecke: Writing - Original Draft, Writing - Review \& Editing, Validation, Data Curation. Laura Kaltwasser: Conceptualization, Investigation, Writing - Review \& Editing. Andrea Hildebrandt: Conceptualization, Methodology, Supervision, Writing - review \& editing.

\section{Declaration of competing interests}

None. The sponsors have no involvement in the study design, data collection, analysis and interpretation of the data as well as writing the report and the decision to submit the article for publication.

\section{References}

Adams, D. C., Collyer M. L., \& Kaliontzopoulou A. (2018). Geomorph: Software for geometric morphometric analyses. R package version 3.0.6. https://cran.r-project.org/package=geomorph.

Baltrusaitis, T., Zadeh, A., Lim, Y.C., \& Morency, L.P. (2018, May). OpenFace 2.0: Facial behavior analysis toolkit. Automatic face \& gesture recognition (FG 2018), 2018 13th IEEE international conference on (pp. 59-66). IEEE.

Banks, G.C., Batchelor, J.H., \& McDaniel, M.A. (2010). Smarter people are (a bit) more symmetrical: A meta-analysis of the relationship between intelligence and fluctuating asymmetry. Intelligence, 38, 393-401. 
Bigoni, L., Krajíček, V., Sládek, V., Velemínský, P., \& Velemínská, J. (2013). Skull shape asymmetry and the socioeconomic structure of an early medieval central European society. American Journal of Physical Anthropology, 150, 349-364.

Borráz-León, J.I., \& Cerda-Molina, A.L. (2015). Facial asymmetry is negatively related to assertive personality but unrelated to dominant personality in men. Personality and Individual Differences, 75, 94-96.

Carr, E. W., Korb, S., Niedenthal, P. M., \& Winkielman, P. (2014). The two sides of spontaneity: Movement onset asymmetries in facial expressions influence social judgments. Journal of Experimental Social Psychology, 55, 31-36.

Claes, P., Walters, M., Vandermeulen, D., \& Clement, J.G. (2011). Spatially-dense 3D facial asymmetry assessment in both typical and disordered growth. Journal of Anatomy, 219, 444455.

Claude, J. (2008). Morphometrics with R. Springer.

Dryden, I.L., \& Mardia, K.V. (1998). Statistical analysis of shape. Chichester: John Wiley \& Sons.

Farkas, L.G., \& Cheung, G. (1981). Facial Asymmetry in health North American Caucasians: An anthropometrical study. The Angle Orthodontist, 51, 70-77.

Fink, B., Grammer, K., \& Thornhill, R. (2001). Human (Homo sapiens) facial attractiveness in relation to skin texture and color. Journal of Comparative Psychology, 115, 92-99.

Fink, B., Neave, N., Manning, J.T., \& Grammer, K. (2005). Facial symmetry and the 'big-five' personality factors. Personality and Individual Differences, 39, 523-529.

Fink, B., Neave, N., Manning, J.T., \& Grammer, K. (2006). Facial symmetry and judgements of attractiveness, health and personality. Personality and Individual Differences, 41, 491-499.

Gangestad, S.W., \& Thornhill, R. (1997). The evolutionary psychology of extrapair sex: The role of fluctuating asymmetry. Evolution and Human Behavior, 18, 69-88.

Garver-Apgar, C.E., Gangestad, S.W., \& Thornhill, R. (2008). Hormonal correlates of women's mid- 
cycle preference for the scent of symmetry. Evolution and Human Behavior, 29, 223-232.

Grammer, K., \& Thornhill, R. (1994). Human (Homo sapiens) facial attractiveness and sexual selection: The role of symmetry and averageness. Journal of Comparative Psychology, 108, 233-242.

Grammer, K., Fink, B., Møller, A.P., \& Manning, J.T. (2005). Physical attractiveness and health: Comment on Weeden and Sabini (2005). Psychological Bulletin, 131, 658-661.

Grewe, C.M., Le Roux, G., Pilz, S.K., \& Zachow, S. (2018, May). Spotting the details: The various facets of facial expressions. Automatic face \& gesture recognition (FG 2018), 2018 13th IEEE International Conference on (pp. 286-293). IEEE.

Grewe, C. M., \& Zachow, S. (2016, October). Fully automated and highly accurate dense correspondence for facial surfaces. European conference on computer vision (pp. 552-568). Springer, Cham.

Hammond, P., Hutton, T.J., Allanson, J.E., Campbell, L.E., Hennekam, R., Holden, S., Patton, M.A., Shaw, A., Temple, K., Trotter, M., Murphy, K.C. \& Winter, R.M. (2004). 3D analysis of facial morphology. American Journal of Medical Genetics Part A, 126, 339-348.

Hildebrandt, A., Sommer, W., Schacht, A., \& Wilhelm, O. (2015). Perceiving and remembering emotional facial expressions - A basic facet of emotional intelligence. Intelligence, 50, 52-67.

Hooda, S., \& D’Souza, M. (2012). Evaluation of facial asymmetry using digital photographs with computer aided analysis. Journal of Indian Prosthodontic Society, 12, 8-15.

Hope, D., Bates, T., Penke, L., Gow, A.J., Starr, J.M., \& Deary, I.J. (2011). Fluctuating asymmetry and personality. Personality and Individual Differences, 50, 49-52.

Horn, J.L. (1965). A rationale and test for the number of factors in factor analysis. Psychometrika, 30, $179-185$.

Hönekopp, J., Bartholomé, T., \& Jansen, G. (2004). Facial attractiveness, symmetry, and physical fitness in young women. Human Nature, 15, 147-167. 
Kaltwasser, L., Hildebrandt, A., Wilhelm, O., \& Sommer, W. (2016). Behavioral and neuronal determinants of negative reciprocity in the ultimatum game. Social Cognitive and Affective Neuroscience, 11, 1608-1617.

Kaltwasser, L., Mikac, U., Buško, V., \& Hildebrandt, A. (2017). No robust association between static markers of testosterone and facets of socio-economic decision making. Frontiers in Behavioral Neuroscience, 11.

Klingenberg, C.P., Barluenga, M., \& Meyer, A. (2002). Shape analysis of symmetric structures: Quantifying variation among individuals and asymmetry. Evolution, 56, 1909-1920.

Ko, E.W.C., Huang, C.S., \& Chen, Y.R. (2009). Characteristics and corrective outcome of face asymmetry by orthognathic surgery. Journal of Oral and Maxillofacial Surgery, 67, 2201-2209.

Koehler, N., Simmons, L.W., Rhodes, G., \& Peters, M. (2004). The relationship between sexual dimorphism in human faces and fluctuating asymmetry. Proceedings of the Royal Society of London B: Biological Sciences, 271, 233-236.

Lele, S.R., \& Richtsmeier, J.T. (2001). An invariant approach to statistical analysis of shapes: Interdisiplinary statistics. Chapman \& Hall/CRC Interdisciplinary Statistics Series.

Liu, Y., Schmidt, K.L., Cohn, J.F., \& Mitra, S. (2003). Facial asymmetry quantification for expression invariant human identification. Computer Vision and Image Understanding, 91, 138-159.

Livshits, G., \& Kobyliansky, E. (1989). Study of genetic variance in the fluctuating asymmetry of anthropometrical traits. Annals of Human Biology, 16, 121 -129.

Livshits, G., \& Kobyliansky, E. (1991). Fluctuating asymmetry as a possible measure of developmental homeostasis in humans: A review. Human Biology, 441-466.

Mayer, C., Windhager, S., Schaefer, K., \& Mitteroecker, P. (2017). BMI and WHR are reflected in female facial shape and texture: A geometric morphometric image analysis. PloS ONE, 12 .

Milborrow, S., \& Nicolls, F. (2014, January). Active shape models with SIFT descriptors and MARS. Computer vision theory and applications (VISAPP), 2014 international conference on (Vol. 2, 
pp. 380-387). IEEE.

Mitteroecker, P., Windhager, S., Müller, G.B., \& Schaefer, K. (2015). The morphometrics of "masculinity" in human faces. PLoS One, 10.

Olderbak, S.G., Hildebrandt, A., Pinkpank, T., Sommer, W., \& Wilhelm, O. (2014). Psychometric challenges and proposed solutions when scoring facial emotion expression codes. Behavior Research Methods, 46, 992-1006.

Palmer, A.R., \& Strobeck, C. (1986). Fluctuating asymmetry: Measurement, analysis, patterns. Annual Review of Ecology and Systematics, 17, 391-421.

Parsons, P.A. (1990). Fluctuating asymmetry: An epigenetic measure of stress. Biological Rreviews, $65,131-145$.

Penke, L., Bates, T.C., Gow, A.J., Pattie, A., Starr, J.M., Jones, B.C., Perrett, D.I. \& Deary, I.J. (2009). Symmetric faces are a sign of successful cognitive aging. Evolution and Human Behavior, 30, 429-437.

Pound, N., Lawson, D.W., Toma, A.M., Richmond, S., Zhurov, A.I., \& Penton-Voak, I.S. (2014). Facial fluctuating asymmetry is not associated with childhood ill-health in a large British cohort study. Proceedings of the Royal Society of London B: Biological Sciences, 281, 20141639.

Prokosch, M.D., Yeo, R.A., \& Miller, G.F. (2005). Intelligence tests with higher g-loadings show higher correlations with body symmetry: Evidence for a general fitness factor mediated by developmental stability. Intelligence, 33, 203-213.

R Core Team (2018). R: A language and environment for statistical computing. R Foundation for Statistical Computing, Vienna, Austria. https://www.R-project.org/.

Rhodes, G. (2006). The evolutionary psychology of facial beauty. Annual Review Psychology, 57, 199226.

Richtsmeier, J.T., Burke Deleon, V., \& Lele, S.R. (2002). The promise of geometric morphometrics. American Journal of Physical Anthropology, 119, 63-91. 
Royston, P. (1995). Remark AS R94: A remark on algorithm AS 181: The W-test for normality. Journal of the Royal Statistical Society. Series C (Applied Statistics), 44, 547-551.

Schaefer, K., Fink, B., Grammer, K., Mitteroecker, P., Gunz, P., \& Bookstein, F.L. (2006). Female appearance: Facial and bodily attractiveness as shape. Psychology Science, 48, 187-204.

Scheib, J.E., Gangestad, S.W., \& Thornhill, R. (1999). Facial attractiveness, symmetry and cues of good genes. Proceedings of the Royal Society of London. Series B: Biological Sciences, 266, 1913-1917.

Schlager, S. (2017). Morpho and Rvcg - Shape analysis in R. In Zheng G., Li S., Szekely G. (Eds.). Statistical shape and deformation analysis. Academic Press.

Schmid, K., Marx, D., \& Samal, A. (2008). Computation of a face attractiveness index based on neoclassical canons, symmetry, and golden ratios. Pattern Recognition, 41, 2710-2717.

Shackelford, T., \& Larsen, R. (1997). Facial asymmetry as an indicator of psychological, emotional, and physiological distress. Journal of Personality and Social Psychology, 72, 456-466.

Simmons, L. W., Rhodes, G., Peters, M., \& Koehler, N. (2004). Are human preferences for facial symmetry focused on signals of developmental instability?. Behavioral Ecology, 15, 864-871.

Song, W.C., Koh, K.S., Kim, S.H., Hu, K.S., Kim, H.J., Park, J.C., \& Choi, B.Y. (2007). Horizontal angular asymmetry of the face in Korean young adults with reference to the eye and mouth. Journal of Oral and Maxillofacial Surgery, 65, 2164-2168.

Sulikowski, D., Burke, D., Havlíček, J., \& Roberts, S.C. (2015). Head tilt and fertility contribute to different aspects of female facial attractiveness. Ethology, 121, 1002-1009.

Svegar, D. (2016). What does facial symmetry reveal about health and personality? Polish Psychological Bulletin, 47, 356-365.

Thiesen, G., Gribel, B.F., \& Freitas, M.P.M. (2015). Facial asymmetry: A current review. Dental Press Journal of Orthodontics, 20, 110-125.

Thornhill, R., \& Gangestad, S.W. (2006). Facial sexual dimorphism, developmental stability, and 
susceptibility to disease in men and women. Evolution and Human Behavior, 27, 131-144.

van Dongen, S. (2012). Fluctuating asymmetry and masculinity/femininity in humans: A meta-analysis. Archives of Sexual Behavior, 41, 1453-1460.

van Dongen, S. (2014). Associations among facial masculinity, physical strength, fluctuating asymmetry and attractiveness in young men and women. Annals of Human Biology, 41, 205213.

van Valen, L.V. (1962). A study of fluctuating asymmetry. Evolution, 16, 125-142.

Weeden, J., \& Sabini, J. (2005). Physical attractiveness and health in Western societies: A review. Psychological Bulletin, 131, 635-653.

Windhager, S., Schaefer, K., \& Fink, B. (2011). Geometric morphometrics of male facial shape in relation to physical strength and perceived attractiveness, dominance, and masculinity. American Journal of Human Biology, 23, 805-814.

White, J.D., Ortega-Castrillón, A., Matthews, H., Zaidi, A.A., Ekrami, O., Snyders, J., \& Claes, P. (2019). MeshMonk: Open-source large-scale intensive 3D phenotyping. Scientific Reports, 9, 111.

Yin, L., Wei, X., Sun, Y., Wang, J., \& Rosato, M.J. (2006). A 3D facial expression database for facial behavior research. 7th international conference on automatic face and gesture recognition, 211216

Zachow, S. (2015). Computational planning in facial surgery. Facial Plastic Surgery, 31, 446-462. 